\title{
La importancia del diagnóstico estratégico en las organizaciones
}

\author{
The importance of the strategic \\ diagnostic in organizations
}

DOI: https://doi.org/10.17981/econcuc.42.2.2021.Ensy.1

Ensayo Argumentativo.

Fecha de recepción: 30/09/2020

Fecha de devolución: 15/11/2020

Fecha de aceptación: 02/02/2021

Fecha de publicación: 18/02/2021

\section{Valentina Arrieta Jiménez \\ Cuidar Salud Barranquilla SAS / \\ Universidad de la Costa \\ Barranquilla, Atlántico (Colombia) \\ valentinaarrieta13@gmail.com}

\section{Yulieth Estela Cervantes Borrero}

Sinergia global en salud SAS /

Universidad de la Costa

Barranquilla, Atlántico (Colombia) yuly2412@hotmail.com

\section{Lina Marcela De la Cruz Lara \\ Fondo de Empleados Av Villas / \\ Universidad de la Costa \\ Barranquilla, Atlántico (Colombia) \\ lincruz2014@gmail.com}

\section{Dayana Marcela López Cadena \\ Ladrillera Barranquilla Ltda / \\ Universidad de la Costa \\ Barranquilla, Atlántico (Colombia) \\ dayanamarcela94@hotmal.com}

Para citar este artículo:

Arrieta, V., Cervantes, Y., De la Cruz, L. \& López, D. (2021). La importancia del diagnóstico estratégico en las organizaciones. Económicas CUC, 42(2), 243-254. DOI: https://doi.org/10.17981/ econcuc.42.2.2021.Ensy.1

\section{Resumen}

El diagnostico estratégico se realiza en todas las empresas, para saber evaluar la situación de la organización, lo cual incluye el análisis dentro y fuera de la organización, es decir interno y externo. El éxito de las organizaciones resulta de la ejecución de estrategias adecuadas y así evitar gastar tiempo, recursos en estrategias innecesarias para su sector de mercado. El diagnostico se realiza con la finalidad de identificar las oportunidades, amenazas, fortalezas y debilidades de la organización. Como objetivo principal identificaremos los aspectos importantes que debe tener en cuenta una organización para realizar un diagnóstico estratégico y de esta manera determinar la importancia de este. La metodología para utilizar consta de investigación en los diferentes autores que nos aporten información relevante con nuestro enfoque para realizar el ensayo y de esta manera llegar como equipo a las conclusiones de la importancia del diagnóstico estratégico en las organizaciones.

Palabras clave: Diagnostico; estrategias; DOFA; organización; entorno; diagnostico estratégico

\begin{abstract}
The strategic diagnosis is carried out in all companies, to know how to evaluate the situation of the organization, which includes the analysis inside and outside the organization, that is, internal and external. The success of organizations results from the execution of appropriate strategies and thus avoid wasting time and resources on unnecessary strategies for their market sector. The diagnosis is carried out in order to identify the opportunities, threats, strengths and weaknesses of the organization. As the main objective we will identify the important aspects that an organization must consider carrying out a strategic diagnosis and thus determine the importance of this. The methodology to be used consists of research in the different authors who provide us with relevant information with our approach to carry out the test and in this way reach as a team the conclusions of the importance of strategic diagnosis in organizations. Keywords: Diagnosis; strategies; SWOT; organization; environment; strategic diagnosis
\end{abstract}




\section{INTRODUCCIÓN}

El ser humano como ser pensante, con capacidad de invención, ha logrado a través del tiempo analizar el entorno de la organización internamente, es decir, los problemas que existan dentro de ella, sus potenciales, hasta dónde puede llegar; el diagnostico estratégico busca la eficiencia de la organización a través de los cambios, procesa la información sobre su entorno, con la finalidad de identificar las oportunidades, amenazas, fortalezas y debilidades de la organización, en vista de esto es necesario conocer la importancia del diagnóstico estratégico en las organizaciones.

Las empresas en la búsqueda de oportunidad dentro del entorno cambiante han avanzado en los últimos años para fortalecer sus estrategias, elevar sus ingresos y generar una estabilidad a sus empleados.

De esta manera la importancia de un buen diagnóstico estratégico en las organizaciones es conocer sus capacidades y a lo que se enfrentan; teniendo en cuenta la economía globalizada y el ambiente empresarial complejo de la actualidad.

La globalización económica son los diferentes fenómenos de creciente que atraviesa la economía por la variedad de factores en lo relacionado con los intercambios de bienes, servicios, capital, tecnología y recurso humano. Barberá (2012) lo define como resultado del deseo de los representantes económicos que buscan aumentar actividades a lo largo del mundo a través de la libertad de movimientos de los diferentes factores, lo que quiere decir que las decisiones que se adopten en uno, de alguna forma afectan o repercuten en otro.

\section{Desarrollo}

\section{Economía, crecimiento y otros elementos de la dirección estratégica}

Según Drucker (1997), a nivel mundial la economía ha presentado un crecimiento en las últimas cuatro décadas de manera acelerada que en cualquier otra época desde la Revolución industrial del siglo XVIII. En donde se reconoce la intervención de varios factores; donde el más significativo ha sido el cambio de estructura de los mercados, que se manifiesta a través de la inauguración de los mercados de las grandes potencias a nivel mundial; otra señal que se presentó fue cuando las grandes potencias empezaron a inducir a economías como Brasil, India y China, con el fin de hacerlas participar en el nuevo contexto mundial, por ejemplo acceder a suministrar mano de obra a bajo costo y aceptar abrir sus mercados a los productos de las grandes corporaciones norteamericanas y europeas, dando forma a un gran socio comercial.

Por lo anterior podemos decir que el diagnostico estratégico le permite a la organización tomar las decisiones adecuadas teniendo en cuenta la variabilidad en la economía a raíz de la globalización (Morstadt y Bello, 2018), siendo esto un impacto de gran peso porque de acuerdo con la razón de ser de la organización, puede que se vea afectada por los factores externos que dependen de su estrategia, como por ejemplo el aumento de precios de las maquinarias, los insumos, entre otros. 
Por otro lado, para Stiglitz (2002), Premio Nobel de Economía en el 2001, la globalización está en término entre bueno y mala, es más que todo como se está gestionando y de qué modo; su opinión, los útiles adversarios de las propia, aumento de la clase baja, incremento de las diferencias, excepción nacional, paro, contaminación, etc. alcanzan a mitigar entre la política económica y es indispensable la existencia de la voluntad política al respecto, recurso muy escaso en los tiempos que corren.

De este modo, Stiglitz (2002) se sinceró a raíz en cuanto a que confía en que el modelo económico vigente el neoliberal es competente de finalizar con la clase baja y la desigualdad social, eternamente y cuando viva de un beneficio político evidente y se den, de igual forma, una cadena de situaciones que admitan una mayor transparencia y contribución ciudadana en las cuestiones públicas (Morstadt \& Bello, 2018; Solano, 2019). Stiglitz (2002) no abandona de entender en la globalización económica a modo de un cambio diferente, y todo en todo instante desde una planta reformador e institucionalista, para entender es, diseñando como excelente camino de una innovación de las instituciones y objetando cualquier actitud más fundamental que admita el establecimiento de un régimen alternativo al de una conveniente globalización.

Lo anterior nos indica que todas las organizaciones deben tener un proceso de adaptación al medio presentado por la llegada de la globalización para así de esta manera llegar aún diagnostico estratégico acertado, siendo capaz de identificar las fortaleza y debilidades que presenta el ambiente.

La puesta en marcha de una estrategia global le implica a la empresa moverse dentro de un esquema donde se integren y regularicen las decisiones estratégicas para alcanzar identidad y vender en todo el mundo donde exista demanda significativa de los compradores (Thompson y Strickland, 2004).

Por otro lado, si el ambiente llegase a presentar complicaciones las cuales pueden ser cambios que se presenten en los cambios tecnológicos, sociales, políticos, entre otros, la organización se encuentra obligada según lo que indica el autor Garrido (2006) al presentar según el nivel de importancia un análisis estratégico partiendo de los elementos faltantes enfatizando a partir de ellos, si es viable al gerente para poder operar con una investigación orientada a la toma de decisiones para la operación o una estrategia.

En referencia a lo antes expuesto, Garrido (2006) expone la importancia de precisar los elementos del diagnóstico estratégico destacando que a partir de ellos le es posible al gerente manejar información orientada a la toma de decisiones para la acción; es decir, sobre la base de la información recopilada a través de estos elementos, es posible concretar las acciones que aporten mayor impulso, considerando las fuerzas que apoyan o derivan dificultades tanto desde el interior de la organización como en su exterior.

\section{Organización y diagnóstico estratégico}

Se puede decir que las organizaciones surgen a partir de coordinar dos contingencias, y estas son la contingencia de reglamentos y normas que la organización ha establecido para regular los comportamientos de los miembros y en la contingencia de los compartimientos de los miembros (Rodríguez, 1999). 
El diagnostico estratégico ayuda a detectar la situación real de la organización y así tomar las medidas correctas para la mejora continua. Se evalúan la efectividad de las estrategias que se planteen y así garantizar que va por el camino correcto, ya que se cuenta con información que ayuda a la toma de decisiones.

Para hacer efectivas las estrategias se debe poner en práctica mediante planes y aumentar su grado de detalle hasta llegar a los aspectos más esenciales de las operaciones. En definición se puede decir que las tácticas son los planes de acción a través del cual se ejecutan las estrategias.

El profesor Porter (1980) ha indicado que la formulación de una estrategia requiere evaluar el atractivo de una industria mediante el análisis del medio ambiente externo. Hay que centrarse en el tipo de competencia de la industria, la posibilidad de que nuevas empresas ingresen en el mercado, la disponibilidad de productos y servicios sustitutos y las perspectivas de convenios de los vendedores, de los compradores y de los consumidores.

De acuerdo con Miles, Snow, Meyer y Coleman (1978), las organizaciones también modifican constantemente y perfeccionan el mecanismo por el cual logran sus propósitos y objetivos, reorganizando su estructura, sus relaciones y sus procesos de gestión.

Si hablamos un poco sobre cómo llevar a cabo un objetivo estratégico, es importante que identifiquemos que tan funcional puede servir todo a la habilidad que debe, David, David y David (2014) y Paredes-Chacín (2017) dicen que todo tiene su entrada desde su punto, es decir, va más allá. Por eso, es significativo que se identifique cuál es su misión, los objetivos y sus organizaciones. Esto con el fin de que en representación de que varias veces el entorno real excluye fundamentos que puedan linear el camino expectación, sin embargo una extensión es que por el extremo que logremos rebuscar su propósito no lo vamos a encontrar de hecho se podría decir que es positivo ya que se analizan mejores las actividades y se determinan una posición frente de la misma, logrando la visión estratégica, de hecho se incluyen hacia el futuro caminando en mediano y largo plazo donde lograremos excelente resultados.

La mayoría de organizaciones llevan procesos capaces de adaptarse a los cambios del entorno, deben identificar un ciclo de adaptación ya que cada una tiene una visión empresarial definida, su segmento de mercado, sus clientes objetivos, su mercado objetivo.

Por otra parte, el perfil de la organización es importante ya que es el punto de partida para determinar el lugar en el que se encuentra y hacia dónde quiere llegar. Así determinan la misión de la organización y clarifican la orientación geográfica del negocio, por ejemplo: en dónde debería de operar, además de evaluar la situación competitiva de las organizaciones.

Los integrantes de la organización diseñan el perfil de esta, los cuales determinan hacia dónde va la empresa o su dirección a través de una visión que responda a la pregunta ¿en qué queremos convertirnos? Por lo tanto, deben examinar cuidadosamente sus valores, preferencias y actitudes, porque ellos repercuten en la estrategia ejemplo: cuando esta la alternativa de vender cigarrillos pudiera parecer rentable, los ejecutivos de la organización pueden decidir contra a la estrategia debido a que en su sistema de valores condena los cigarrillos. 
El éxito empresarial exige la capacidad de reaccionar y adaptarse a situaciones imprevistas del entorno, es importante realizar monitoreos sobre la ejecución de los planes frente a estos cambios, que como resultado podrían llevar a identificar y generar mejoras que permitirían plantear objetivos estratégicos que ayuden a un excelente desempeño de la organización, para así lograr el cumplimiento de los objetivos establecidos por la organización que hayan sido identificados, de tal forma, que se lleven a cabo las herramientas necesarias, que sirvan ante las situaciones inesperadas como facilitadores para mantenerse en la competencia.

En el diagnostico estratégico organizacional es de vital importancia validar o realizar un análisis del entorno, es decir aquellos elementos o factores que rodean la organización, así como de igual forma es vital el análisis interno, como, por ejemplo, sus fortalezas, oportunidades, amenazas y debilidades. Este análisis es complejo y está sometido a cambios constantemente.

Las organizaciones frente a estos problemas pueden tomar diferentes posiciones pueden ser pasivos con mucha posibilidad de seguir estos cambios, o se adaptan a la nueva situación y contribuye activamente a estos cambios.

El análisis DOFA/FODA ayuda a identificar cuáles son los puntos fuertes y debilidades que tiene la organización es decir el análisis interno de ella, las oportunidades y amenazas que hacen parte del análisis externo.

Thompson y Strikland (1998) indican que el análisis FODA considera o aprecia el efecto que una estrategia tiene para lograr un equilibrio entre las capacidades que la organización posee y su situación externa, es decir, las oportunidades y amenazas.

Prieto (2008) y Sukier et al. (2020) comentan que las fortalezas son situaciones, hechos, fenómenos, eventos internos que establecen en la organización una notable ventaja competitiva; es decir es lo que le conviene, sus puntos fuertes de la organización y le ayudan al logro de objetivos. Por su parte, Münch (2005) plantea que las debilidades son factores propios de la empresa que impiden el logro de los objetivos, lo cual es fundamental para poder emprender con mayor seguridad y así generar transformaciones importantes.

Las oportunidades y amenazas son esas variables que la empresa encuentran en el ambiente exterior las cuales no pueden ser controladas por la empresa, las cuales impactan ya sea de manera positiva o negativa en el desarrollo de la empresa, por tal razón es importante estar alertas a lo que, en los diferentes sectores de la economía, tecnología, política y la sociedad en general.

Para Porter (1998), las fortalezas y oportunidades son, en conjunto, las capacidades, esto es, los aspectos fuertes como débiles de las organizaciones o empresas competidoras (productos, distribución, comercialización y ventas, operaciones, investigación e ingeniería, costos generales, estructura financiera, organización, habilidad directiva, etc.).

A través de la matriz DOFA diferentes organizaciones han podido esquematizar los factores internos más importantes (fortalezas y debilidades) y externos (oportunidades y amenazas) que pueden impactar su futuro (Kangas, Kurttila, Kajanus \& Kangas, 2003). Las fortalezas y debilidades conforman un entorno interno que puede ser controlado, mientras que los otros factores están en un ambiente externo que no puede 
ser manipulado (Houben, Lenie \& Vanhoof, 1999). Con los factores mencionados, los gerentes pueden determinar cuatro tipos de estrategias: de fuerzas y debilidades, de debilidades y oportunidades, de fuerzas y amenazas, y de debilidades y amenazas (Weihrich, 1982; Sukier et al., 2020).

\section{Diagnóstico estratégico y las estrategias}

Se puede decir que este análisis FODA es uno de los planificadores que emplean las organizaciones que con el fin de lograr y contribuir con las decisiones que fueron identificadas a través de las estrategias; FODA se puede definir como una pieza clave y fundamental a la hora de tomar decisiones, que más que una herramienta, es un método de importancia que del mismo modo ayuda a la identificación y aporta numerosas estrategias que son vitales en las decisiones que toman las organizaciones, de igual manera es de gran importancia observar las debilidades y fortalezas a la hora de realizar el análisis FODA, así orientamos a generar una ventaja y preparación frente a cambios en el ambiente, por la estrategia buscamos una buena planeación.

Las estrategias representan los planes o acciones que la empresa piensa ejecutar con el fin de ir acorde con su misión y lograr sus objetivos, las cuales son el resultado de un análisis realizado e identificado a través del FODA y que permite identificar necesidades de la organización para así plantear los objetivos que van a ser utilizados por la empresa para lograr las metas propuestas.

Según Gómez-Mejía y Balkin (2003), "el método de un diseño o el camino hacia el método, parte de la formulación de una estrategia que logre alcanzar la misión de una organización" (p. 159). Una estrategia completa ordenada y basada en todos los recursos y capacidades que posee la empresa será una gran oportunidad de destacar la organización sobre sus competidores.

Para David (1995), anunciar el proceso consiste en identificar las partes que la componen como aquellos factores que intervienen tanto en lo interno como en lo externo, hacen parte de la formulación de la estrategia, con el fin de lograr la creación de objetivos que permitan formular estrategias y seleccionar las mejores o aquellas que se consideran las más apropiadas para trabajar y aplicar a la empresa. De acuerdo con Wheelen y Hunger (2007), administrar las oportunidades y amenazas ambientales de una manera eficaz, haciéndole frente con las fortalezas y las debilidades de la organización permitirán el desarrollo de los planes que se poseen a largo plazo a través de una formulación estratégica.

Dess y Lumpkin (2003) definen que la formulación estratégica tiene a lugar varios niveles: la estrategia a nivel de unidad de negocio; la estrategia a nivel corporativo; las estrategias internacionales; y las estrategias de Internet y de e-business. Según lo anterior existen diferentes tipos de estrategias que son utilizadas por múltiples organizaciones con el fin de destacarse ante las exigencias del mercado, siendo el punto de referencia de los consumidores, creando un valor agregado que sitúe a la organización por encima de sus competidores y que en la actualidad puede lograrse a través del direccionamiento estratégico. 


\section{- Estrategias corporativas}

Las estrategias corporativas para Hill y Jones (2005) es aquella que "resuelve" las preguntas fundamentales como: ¿en qué negocio o negocios debemos estar para maximizar la rentabilidad de la organización a largo plazo? y ¿de qué manera se debe dar a conocer la organización para generar un aumento de su presencia en el mercado para así generar ventaja competitiva?".

Collis y Montgomeri (2007) mencionan las estrategias corporativas como "el método en que la organización genera valor a través de la coordinación de las actividades de los diferentes mercados". Es decir, las organizaciones no sólo deben enfocarse a una sola estrategia, por el contrario, deben ampliar su visión, sin delimitar su negocio y apuntándole al crecimiento internacional de la misma.

Según Hitt, Ireland y Hoskisson (2004), las estrategias internacionales: expresan que "la estrategia internacional implica que la empresa venda sus bienes o servicios fuera de su mercado nacional". La ventaja competitiva debe llevarse a un nivel internacional, pues el mercado es amplio y los bienes o servicios pueden satisfacer las necesidades de consumidores que se encuentran más allá de cualquier frontera.

Hay diferentes razones por la que una empresa decide expandirse a nivel internacional, un reconocimiento de esta categoría le permitirá incursionar en mercados mundiales logrando así, un aumento de sus activos e ingresos, posición, estabilidad, aumento en las inversiones de productos y proceso, experiencia y sobre todo esa tan anhelada ventaja competitiva en el mercado.

\section{- Las estrategias en Internet:}

El uso adecuado de las estrategias tecnológicas y de e- commerce les permite a las organizaciones mejorar en diferentes áreas sobre todo en la tecnológica aumentando sus capacidades y su nivel competitivo. Dess \& Lumpkin (2003) anuncian que en la organización no es importante la tecnología como tal, sino el uso en cómo se le da para lograr obtener transacciones rentables que permitan destacar su negocio.

La tecnología es cambiante y cada vez más innovadora, la cual se ha convertido en un elemento fundamental en el ámbito empresarial, por tal razón las organizaciones deben aprender a acomodar sus estrategias basadas en niveles tecnológicos que faciliten el buen uso de estas y les permita a las empresas romper barreras, resolver problemas, facilitando el crecimiento de las organizaciones y su nivel de competitividad.

Es de resaltar que Vargas y Guillén (2004) enfatizan en la necesidad de las organizaciones de crear estrategias para permanecer en el mercado, y también resaltan la función del estratega para el logro de este objetivo. Burbano (2011) afirma que la complejidad al definir una estrategia resulta estar presente en la implementación de los objetivos ya que estos deben ser claros enfocado en la meta y muy importante que deben estar relacionado con el presupuesto.

Por otro lado, centraliza que la implementación de una estrategia no se basa en llevar a cabalidad un paso a paso matemático inmerso en formulas; antes bien es de gran importancia la participación de los directivos y sus colaboradores tanto interno como externo para el logro de los objetivos. 
De acuerdo con lo anterior se puede inferir, que si una organización incurre en el involucramiento de todo su equipo de trabajo este lograría cumplir sus estrategias, porque al compartirlas todos tendrán un mismo foco para el desarrollo de los objetivos planteados.

Como también realimentar al equipo le permite a la organización mantener su cadena valor y sin dejar a un lado los indicadores financieros los cuales deben ser medidos para determinar el avance y generar planes de acción.

Thompson, Peteraf, Gamble y Stickland (2018) y Pérez, Chumaceiro y Acosta (2019), exponen que la estrategia de una organización es en parte proactiva y en parte reactiva; lo anterior se puede relacionar con la importancia del direccionamiento estratégico ya que si tenemos en cuenta en el direccionamiento las acciones proactivas para el aumento de los resultados financieros de la organización y la estabilidad de las méritos competitivos, los cuales serán el punto de partida para generar planes de acción que le permitan a la organización tomar las mejores decisiones.

Cuando hablamos de reactividad esta es vista cuando se realiza análisis del entorno externo por lo general son manejados por estrategias experimentales, con métodos de prueba y error que a su vez nos llevan a terminar en un plan proactivo. Es decir, que existe una sinergia continua entre la proactividad y la reactividad en el manejo de las estrategias organizacionales.

\section{Responsabilidad social como parte del direccionamiento estratégico}

Las organizaciones poco a poco han evolucionado y se van adaptando al entorno donde cada vez presenta mayor eficacia, los mercados y las necesidades son cada vez más complejos, y las cuales pueden ser socialmente responsables.

Lo cual no depende solo de su tecnología, de sus procesos de producción ni de su gestión financiera, depende del respeto por el cuidado del medio ambiente, de las personas alrededor y de los empleados de la organización.

La norma ISO 26000 (2010) se propone que la característica e la responsabilidad social es decisión de las organizaciones dar importancia a las consideraciones ambientales y sociales en su toma de decisiones y así rendir cuentas por los impactos de sus decisiones y actividades en el medio ambiente y la sociedad.

Vargas (2014) define la responsabilidad social empresarial como un conjunto de responsabilidades que la empresa se hace cargo de manera voluntaria como resultado de demandas y expectativas de las partes interesadas o stakeholders. Estas responsabilidades no son obligatorias, es decir la organización puede implementarla de manera voluntaria, ya que no forman parte de las normas legales si no en las demandas de accionistas, trabajadores, clientes y medios de comunicación.

Las definiciones anteriormente mencionadas coinciden en varios elementos, en tanto la responsabilidad social empresarial se entiende como el conjunto de compromisos que trascienden la dimensión legal y que, de manera voluntaria, asume una organización empresarial con todos los actores, internos y externos, que tienen interés en la misma (Cubillos, Montealegre y Delgado, 2016; Archibold, Aguilera y Escobar, 2017; Guerrero, 2019; Barragán, García-Guiliany, Meza-Rodríguez, Mercado y Olarte, 2020). 
La Responsabilidad Social (RSE) se podría definir como el compromiso que tienen los miembros de una sociedad, es la contribución al desarrollo sostenible, comprometido hacia la mejora de la calidad de vida de las personas (Pérez et al., 2019). También se puede definir como herramienta de ventajas en la calidad de sus trabajadores, en la cual se puede lograr un buen clima laboral.

Si los empleados se sienten felices con su trabajo, los resultados serán positivos para la organización.

Para Bateman y Snell (2005), "la empresa socialmente responsable maximiza los efectos positivos sobre la sociedad y minimiza sus efectos negativos" (p. 147), es decir que sus aspectos a mejorar disminuyan y los beneficios aumenten.

La RSE es una nueva inclinación de cambio en las organizaciones, por lo deben ser acorde con el desarrollo del entorno que rodea, lo cual se convierte en foco de crecimiento para la sociedad, que permita a las organizaciones ser sustentables y eficientes.

Las organizaciones del entorno deben apropiar y asociar a su cultura a la RSE. Incluyendo las políticas, estrategias y acciones que estas prácticas requieren, y de igual manera, y tomando en cuenta su actividad como forma de divulgación a la comunidad, estas políticas, estrategias y acciones pueden resultar con una mayor efectividad, debido esto, por supuesto, a la masividad y el alcance de estas organizaciones (Barragán et al., 2020).

Según indica Rodríguez-Guerra y Ríos-Osorio (2016) en orientación al desempeño ambiental según su origen de gestión en sus materiales, agua, energía, emisiones, transporte, cumplimientos regulatorios (Archibold et al., 2017; Solano, 2019), vertimientos, biodiversidad y residuos. De igual forma los programan de ambientales desarrollan que cumplan con minimizar todo a aquello impacto negativo que genera su pertinente acción productiva. Las organizaciones tienen un ángulo de valoraciones por un estudio todo analizan tipo de habilidad hacia la falta de acciones más contundentes, del mismo modo se realizan propuesta para que las organizaciones empiecen procedimientos en relación a la sostenibilidad (Pérez et al., 2019). De manera análoga (Rodríguez-Guerra \& Ríos-Osorio, 2016; Ramírez, Chacón y Valencia, 2018), en relación a el desempeño social de las organizaciones valoradas gestionan talento humano, ocupacional, políticas RSE e interacción con comunidades entre otras; las compañías fundadas bajo la guía de SBDC requiere de un buen trabajó de $\mathrm{RH}$, económico y físico para iniciar. La microempresa busca conocimiento vivido y como está construida en empleos nuevos ya que son un objetivo claves según los métodos de responsabilidad social y ambiental alguna representación evidencia el bajo nivel de adelanto en los métodos antes mencionados.

\section{Conclusiones}

El diagnostico estratégico es una herramienta fundamental que deben implementar las organizaciones con el fin de buscar la eficiencia a través del conocimiento de su entorno, esta determina aspectos claves logrando así identificar las oportunidades y amenazas a las que se enfrenta, de tal manera que se puedan fortalecer sus debilidades y aumentar sus fortalezas que le permitan alcanzar sus objetivos y metas propuestas a través 
de las diferentes estrategias planteadas. Este proceso a lo largo de cada etapa será la base de las diferentes perspectivas de crecimiento empresarial y del impacto que pueda generar la organización en las exigencias del mercado y su economía globalizada.

La formulación de las estrategias permitirá a la organización crear esa táctica o acción con la que decida enfrentarse en el mercado que le ayudará a analizar sus propósitos a través del estudio obtenido en base al análisis interno y externo realizado. Esto con el fin de poder impactar el mercado a través de su crecimiento empresarial, generando competitividad, valor y una gran oportunidad de expandirse a nivel internacional logrando el reconocimiento y la posición que desea, así mismo es importante destacar que para permanecer en el mercado es vital la función que cumple el estratega a lo largo de sus funciones.

\section{Reconocimientos y Agradecimientos}

Este ensayo es producto de proyecto de aula de la asignatura Direccionamiento Estratégico adscrita al área de conocimiento de organizaciones del Programa de Administración de Empresas del Grupo de Investigación: Administración Social de la Universidad de la Costa.

Proyecto de aula orientado por la profesora Ana Cecilia Chumaceiro Hernández.

\section{REFERENCIAS}

Archibold, W., Aguilera, L. y Escobar, A. (2017). Revisoría fiscal y sostenibilidad empresarial en Colombia. Económicas CUC, 38(2), 77-88. https://doi.org/10.17981/ econcuc.38.2.2017.06

Barberá, R. (2012). Globalización. En, J. Malfeito (Coord.), Introducción a la economía mundial. [pp. 467-491]. Madrid: Delta Publicaciones.

Barragán, C., García-Guiliany, J., Meza-Rodríguez, V., Mercado, M. y Olarte, L. (2020). Responsabilidad social empresarial en medios impresos en los Departamentos del Atlántico y Magdalena (Colombia). Económicas CUC, 41(1), 187-202. https://doi. org/10.17981/econcuc.41.1.2020.Org.3

Bateman, T. S. y Snell, S. A. (2005). Administración: Un nuevo panorama competitivo. (4 ed.). México, D.F.: McGraw-Hill/Interamericana.

Burbano, J. E. (2011). Presupuestos: un enfoque de direccionamiento estratégico, gestión, y control de recursos (4 ed.). Bogotá, D.C.:McGraw-Hill.

Collis, D. J. y Montgomeri, C. (2007). Estrategia corporativa un enfoque basado en los recursos (2 Ed.). India: McGraw-Hill.

Cubillos, C., Montealegre, J. y Delgado, A. (2016). Responsabilidad social empresarial y stakeholders en organizaciones de tamaños y actividades diferentes. Económicas CUC, 37(2), 115-136. https://doi.org/10.17981/econcuc.37.2.2016.06

David, F. R. (1995). Concepts of strategic management. New Jersey: Prentice Hall.

David, M. E., David, F. R. \& David, F. R. (2014). Mission statement theory and practice: A content analysis and new direction. International Journal of Business, Marketing, \& Decision Science, 7, 95-110. Available: https://www.strategyclub. com/wp-content/uploads/2014/11/MissionArticle.pdf-file.pdf 
Dess G. G. y Lumpkin G. T. (2003). Dirección estratégica: creando ventajas competitivas. Madrid: McGraw-Hill.

Drucker, P. (1997). Visión sobre la administración, La organización basada en la información, la economía, la sociedad. Bogotá, D.C.: Norma.

Garrido, S. (2006). Dirección Estratégica. Madrid: McGraw-Hill/Interamericana.

Gómez-Mejía, L. R. y Balkin B. D. (2003). Administración. Madrid: McGraw-Hill/ Interamericana.

Guerrero, A. (2019). Responsabilidad social y ambiental, análisis para tres microempresas en Ipiales, Colombia. Económicas CUC, 40(2), 53-69. https://doi.org/10.17981/ econcuc.40.2.2019.04

Hill, Ch. W. y Jones, G. R. (2005). Administración estratégica, un enfoque integrado. (6 ed.). México, D.F.: McGraw-Hill.

Hitt, M. A., Ireland, R. y Hoskisson R. (2004). Administración estratégica: competitividad y conceptos de globalización. México, D.F.: Internacional Thomson Editores.

Houben, G., Lenie, K. \& Vanhoof, K. (1999). A knowledge-based SWOT-analysis system as an instrument for strategic planning in small and medium sized enterprises. Decision Support Systems, 26(2), 125-135. https://doi.org/10.1016/S01679236(99)00024-X

Kangas, J., Kurttila, M., Kajanus, M. \& Kangas, A. (2003). Evaluating the management strategies of a forestland estate--the S-O-S approach. The Journal of Environmental Management, 69(4), 349-358. https://doi.org/10.1016/j.jenvman.2003.09.010

Miles, R., Snow, C., Meyer, A. \& Coleman, H. (1978). Organizational strategy, structure and process. New York: McGraw-Hill.

Morstadt, J. y Bello, M. (2018). Indicadores de impacto social para evaluación de proyectos de vinculación con la colectividad. Económicas CUC, 39(1), 105-116. https:// doi.org/10.17981/econcuc.39.1.2018.07

Münch, M. (2005). Planeación Estratégica. México, D.F.: McGraw-Hill.

Organización Internacional de Normalización. (2010). Guía de responsabilidad social. [ISO 26000]. Disponible en https://www.iso.org/obp/ui\#iso:std:iso:26000:ed-1:v1:es

Paredes-Chacín, J. (2017). Planificación financiera ante la perspectiva organizacional en empresas cementeras del estado Zulia-Venezuela. Económicas CUC, 38(1), 105-132. https://doi.org/10.17981/econcuc.38.1.05

Pérez, M. E., Chumaceiro, A. y Acosta, I. (2019). Sostenibilidad social en el sector agroindustrial del estado Trujillo-Venezuela. Un constructo relevante para el desarrollo. Opción, 35(90), 100-149. Disponible en https://produccioncientificaluz. org/index.php/opcion/article/view/30460/31506

Porter, M. E. (1980). Industry structure and competitive strategy: keys to profitability. Financial Analysts Journal, 36(4), 30-41. Available: https://www.jstor.org/ stable/4478361

Porter, M. E. (1988). Técnicas para el análisis de los sectores industriales y de la competencia. México, D.F.: CECSA.

Prieto, J. (2008). Gestión Estratégica Organizacional. Guía práctica para el diagnóstico empresarial. Bogotá, D.C.: Eco Ediciones. 
Ramírez, R., Chacón H. y Valencia, K. (2018). Gestión del Talento Humano como estrategia organizacional en las pequeñas y medianas empresas. Revista CIGAC, 16(1), 20-42. Recuperado de http://ojs.urbe.edu/index.php/cicag/article/view/2929

Rodríguez, D. (1999). Diagnóstico Organizacional. (3 ed.). México, D.F.: Alfaomega.

Rodríguez-Guerra, L. C. y Ríos-Osorio, L. A. (2016). Evaluación de sostenibilidad con metodología GRI. Dimensión Empresarial, 14(2), 73-89. https://doi.org/10.15665/ rde.v14i2.659

Solano, E. (2019). Estabilidad ocupacional reforzada en Colombia: una mirada crítica desde la jurisprudencia. Jurídicas CUC, 15(1), 47-68. https://doi.org/10.17981/juridcuc.15.1.2019.02

Sukier, H., Ramírez, R., Parra, M., Martínez, K., Fernández, G. \& Lay, N. (2020). Strategic Human Resource Management from a sustainable approach. Opción, 36(91), 929-953. Recuperado de https://produccioncientificaluz.org/index.php/opcion/article/view/32144

Stiglitz, J. (2002). El malestar en la globalización. Madrid: Taurus.

Thompson, A. A. y Strickland, A. J. (2004). Administración estratégica: textos y casos. (13 ed.). México, D.F.: McGraw-Hill.

Thompson, A. \& Strikland, K.F.C. (1998). Dirección y administración estratégicas. Conceptos, casos y lecturas. México, D.F.: McGraw-Hill/Interamericana.

Thompson, A. A., Peteraf, M. A. Gamble, J. y Stickland, A. J. (2018). Administración estratégica. México, D.F.: McGraw-Hill.

Vargas, G. A. (2014). La responsabilidad social empresarial en la construcción de paz: una introducción. Bogotá, D.C.: Universidad de los Andes.

Vargas, J. G. \& Guillén I. J. (2004). Procesos de transformación estratégica en relación con la evolución de las organizaciones. Revista escuela de administración de negocios, (52), 14-27. Disponible en https://journal.universidadean.edu.co/index.php/ Revista/article/view/306

Weihrich, H. (1982). The TOWS matrix-A tool for situational analysis. Long Range Planning, 15(2), 54-66. https://doi.org/10.1016/0024-6301(82)90120-0

Wheelen, T. L. y Hunger, J. D. (2007). Administración estratégica y política de negocios: conceptos y casos. (10 ed.). México, D.F.: Pearson Education.

\section{BIODATA}

Valentina Arrieta Jiménez es estudiante del séptimo semestre de Administración de Empresas. ORCID https://orcid.org/0000-0001-9491-5559

Yulieth Estela Cervantes Borrero es estudiante del séptimo semestre de Administración de Empresas. ORCID https://orcid.org/0000-0002-2511-7876

Lina Marcela De la Cruz Lara es estudiante del séptimo semestre de Administración de Empresas. ORCID https://orcid.org/0000-0001-9186-5904

Dayana Marcela López Cadena es estudiante del séptimo semestre de Administración de Empresas. ORCID https://orcid.org/0000-0001-7718-6606 